\title{
Research on Assessment of Children Educational Products
}

\author{
Aiying Liang*, Wenbin Xiao \\ Guangdong Science Center, Guangzhou, China \\ *Corresponding author: gd_sc@yahoo.com
}

Received November 27, 2014; Revised December 04, 2014; Accepted December 08, 2014

\begin{abstract}
Children's educational products play an important role in enhancing children's thinking, analytical and judgment abilities, flexibilities and creativity. However, so far there have been no effective assessment criteria for such products. The article proposes an innovative 3-level index system to assess educational products, which comprises 3 first-level indices, 9 second-level indices and some corresponding third-level indices on the basis of pedagogic, psychological and socialogical theories. The system puts forth specific requirements for qualified educational products in terms of educational effects and operating rules, etc., and incorporates the dimension of "age" to make assessment more operable and accurate.
\end{abstract}

Keywords: educational products, assessment index, educational effects

Cite This Article: Aiying Liang, and Wenbin Xiao, "Research on Assessment of Children Educational Products.” American Journal of Educational Research, vol. 2, no. 12 (2014): 1199-1202. doi: 10.12691/education2-12-10.

\section{Introduction}

Educational products (hereinafter refer to as "EPs") here mainly refer to the products that help childhood enrichment, to serve both the purposes of education and recreation. Different from toys (e.g. dolls, toy guns, etc.) or educational supplies (e.g. textbooks, stationery, etc.), EPs enable children to be educated and pleased while they are interacting with EPs. Such products help children to cognize the world around, develop physical coordination and shape psychological traits such as creativity, imagination, memory, language skills, will power and cooperative spirits.

Since parents are more and more keen on children's development, there are various types of EPs poping up under strong demands. How the EPs exactly work should be paid more attention to. Some of them are found too simple or misleading in function or content, or even go against the cognitive development theories of children with adverse effect on children's growth. For example, some product boasting of intelligence development misguided, in fact, its target group, the 2-to-3-year-olds to practice puzzles propitious to the 8-to-9-year-olds. Such premature experience could cost the children to miss the key period of proper development of cognitive abilities and make against to their normal growth. Therefore, it is not only conducive to standardization of the EP market but also a truly contributive force in the physical and psychological development of the children to evaluate the design objectives and implementation effects of EPs from the angle of children's education and care.

\section{Index System of EP Assessment}

The target group of EPs is definded as children from new-born to 14 years old. According to children development theories, individual development of children at different ages primarily includes three aspects: physical development, cognitive development and social development. Physical development refers to the growth of physical functional systems and the development of senses of the world around. Cognitive development is mainly reflected in the development of thinking ability, language skills, memory ability, and problem-solving ability. Social development, reflected in both the individual and social aspects, refers to the development of self-consciousness, emotions, personality and various social relationships of the children. Therefore, these three critical aspects are considered particularly important in the proper assessment of EPs. Safety is not discussed in the article because safety is prerequiste for any product and there are compulsory standards to comply with. Besides, in view of the outcomes of the sampling survey on EP producers, educators, parents and children about their opinions of EP, we herein propose a three-level index system for EP assessment (see Table 1).

The first-level indices are the basic and mandatory criteria for EPs and must all be fulfilled. They define EPs in distinct terms to differentiate them from other products: "Education" could distinguish EPs from ordinary toys, "Sociality" could separate EPs from some game products (e.g. video games), and "Gameplay" could tell apart between EPs and study materials such as textbooks, reference books. 
Second-level indices provide specifications as well as assessment guidance for the first-level indices. The second-level indices under a first-level index must all be met at the same time. The third-level indices are designed in view of different age grades to provide specific definitions for the age-related second-level indices. Since age grades are mutually exclusive, it is not required to meet all the third-level indices under an index but meets only one of them. For example, a product targeting kids of aged 3-6 years would be deemed to have fulfilled the second-level index "Cognitive" if it has met the third-level index "3-6years".

Table 1. Assessment Index System for an educational product

\begin{tabular}{|c|c|c|}
\hline $\begin{array}{l}\text { First-level } \\
\text { index }\end{array}$ & $\begin{array}{l}\text { Second-level } \\
\text { index }\end{array}$ & Third-level index \\
\hline \multirow{9}{*}{ Education } & \multirow{4}{*}{ Cognitive } & $0-3$ years old \\
\hline & & 3-6 years old \\
\hline & & 6-11 years old \\
\hline & & 11-14 years old \\
\hline & Informative & No third-level indices are provided. \\
\hline & \multirow{4}{*}{ Creative } & $0-3$ years old \\
\hline & & 3-6 years old \\
\hline & & 6-11 years old \\
\hline & & 11-14 years old \\
\hline \multirow{3}{*}{ Sociality } & $\begin{array}{l}\text { Social } \\
\text { adaptability. }\end{array}$ & \multirow{3}{*}{ No third-level indices are provided } \\
\hline & $\begin{array}{l}\text { Cultural } \\
\text { adaptability }\end{array}$ & \\
\hline & Aesthetic & \\
\hline \multirow{3}{*}{ Gameplay } & Interesting. & \multirow{3}{*}{ No third-level indices are provided } \\
\hline & Pleasurable & \\
\hline & Operable & \\
\hline
\end{tabular}

\section{Index Spcification}

\subsection{Education}

The main index "Education" here means that the EP should be effective in promoting children's ability development. Such abilities are embodied in three categories, that is, "cognitive", "informative" and "creative". As the "cognitive" and the "creative" demonstrate clear and definite characteristics at different age stages, both indices set up third-level indices corresponding to different age groups. However, there are no third-level index is offered for the index "Informative" because knowledge is too comprehensive to provide distinct characteristics at certain age group.

\subsubsection{Cognitive}

The "Cognitive"means that EPs should comply with the cognitive characteristics of children and be effective in boosting their cognitive development. The general cognitive abilities of children include attention, perception, memory, imagination, thinking ability, etc., which are the major targets for EPs. Products such as bicycles, skateboards, etc., which only focus on developing physical strength or training athletic skills, are not up to the requirements of this index and therefore should not be classified as EPs.
Four sub-indices based on age elaborate the "Cognitive” as follows:

\subsubsection{0-3 Years Old}

EPs for this age group mainly serve to develop and train up the sensory abilities of targeted children (hereinafter refer to as "TC") and stimulate them to explore the world around, understand the objects and use symbols. At least one of the following functions should be provided:

1. to exercise TC's perceptions including visual, aural, tactile, gustatory and olfactory etc.

2. to encourage TC to play with simple symbols, such as numbers, letters, patterns, etc.

3. to develop TC's intuitive thinking of action and inspire them to start thinking in an activity or operation.

4. to help TC understand the independence of external objects, for example, an EP to keep hiding and showing an object.

Examples of EPs that meet the requirements for the index:

1. colorful hand shaking toys

2. simple hand-controlled sound- or light-emitting products

Examples of EPs that don't meet the requirement for the index:

1. products which, assembled with small sophisticated pieces, demand complicated finger motions or controls and adjustments;

2. products which require delicate contours or sophisticated colored decorations;

3. products which demand remote-control;

4. products which demand conscious imagination;

5. products which need concrete thinking or abstract thinking, e.g. products that ask TC to think according to imagery, words, mathematical symbols, etc.

\subsubsection{3-6 Years Old}

EPs for this age group mainly serve to teach TC in multiple ways how to apply various symbols, and build up TC's views of other person and the reality with respect for TC's egocentricity and imaginary world. At least one of the following functions should be provided:

1. to develop TC's fine-motor skills, such as complicated finger movements, hand-eye coordination, etc.

2. to help TC understand and apply various symbols, such as, words, numbers, letters, etc.

3. to provide TC materials and guidance for fantasy play;

4. to provide TC materials and guidance for constructive play

5. to develop TC's imagery thinking and enables them to imagine with the help of concrete images.

Examples of EPs that meet the requirements for the index:

1. building blocks

2. remote-controlled toys

3. audio-visual learning devices

Examples of EPs that don't meet the requirements for the index:

1. products which demand TC to understand complicated temporal relations; 
2. products which demand TC to understand complicated spatial relations;

3. products which require TC to engage in complicated logical thinking.

\subsubsection{6-11 Years Old}

EPs for this age group mainly serve to develop and train up TC's logical thinking abilities, with the emphasis on abstract generalization, classification, spatial relations, temporal relations, etc. At least one of the following functions should be provided:

1. to develop TC's meaningful memory ability;

2. to develop TC's conscious imagination;

3. to help TC to learn various concepts;

4. to develop TC to learn classification;

5. to help TC understand spatial relations;

6. to help TC understand temporal relations.

Examples of EPs that meet the requirements for the index:

1. mazes,

2. board games,

3. magic cubes,

4. assembly games.

\subsubsection{11-14 Years Old}

EPs for this age group mainly serve to further develop and train up TC's logical thinking abilities, with the emphasis on deductive, inference, dialectical thinking, etc. At least one of the following functions should be provided:

1. to help TC learn memory strategies;

2. to help TC grasp abstract concepts;

3. to develop TC's abilities of deduction and inference;

4. to develop TC's dialectical thinking;

5. to help TC understand more complicated spatial relations.

Examples of EPs that meet the requirements for the index:

1. complex assembly games.

\subsubsection{Informative}

The "Informative" means that EPs should offer children accurate scientific knowledge that complies with their cognitive development. At least one of the following types of knowledge should be provided:

1. Natural science;

2. Social science;

3. Mathematics;

4. System science

5. Thinking science;

6. Physiological science;

7. Literature and arts;

8. Military science;

9. Behavior science.

Any of the following knowledge should not be included:

1. Knowledge with scientific errors;

2. Knowledge beyond TC's cognitive development (Please refer to the Section 3.1.1 for children's cognitive development of different age groups);

3. Knowledge conveying incorrect values.

\subsubsection{Creative}

The "Creative” means that EPs should enrich children's creativity and stimulate their innovative awareness, based on their cognitive development characteristics and knowledge experience.

Four sub-indices based on age elaborate the "Creative" as follows:

\subsubsection{0-3 Years Old}

Because children aged between $0-3$ are at the preparatory stage of creativity, there is no separate requirement for the sub-index. The sub-index could be assessed according to the "Cognitive" 1.1.1.

\subsubsection{3-6 Years Old}

EPs for this age group mainly serve to protect and stimulate TC's curiosity and develop their creative imagination. At least one of the following functions should be provided:

1. to guide TC to find problems and put forward questions via life experiences;

2. to cultivate TC's creative imagination;

3. to develop TC's intuition.

\subsubsection{6-11 Years Old}

EPs for this age group mainly serve to cultivate divergent thinking and provide guidance for simple creative activities. At least one of the following functions should be provided:

1. to guide TC to find problems and put forward questions during learning;

2. to cultivate TC's creative imagination;

3. to cultivate TC's divergent thinking.

\subsubsection{11-14 Years Old}

EPs for this age group mainly serve to teach TC creation techniques and guide some creative activities at certain degrees of difficulty. At least one of the following functions should be provided:

1. to guide TC to find problems and put forward questions during learning and social activities;

2. to help TC learn and grasp creation techniques in an active manner;

3. to prompt TC to complete creative activities at certain degrees of difficulty.

\subsection{Sociality}

The main index "Sociality" means that EPs should promote social psychology development of children to shape proper self-awareness and social consciousness, and help them build up favorable social relationships and actively adapt to the social and cultural environment.

The "Sociality" contains thereunder three categories, among which the two indices of "Social adaptability" and "Cultural adaptability” are organically related. Social adaptability is the core of social development whereas cultural adaptability is the underlying background for social development. The index "Aesthetic" is relatively independent while is essential for product design. Furthermore, aesthetics itself is certainly a social concept. That is why it is categoried under the index "Sociality". The three second-level indices of the "Sociality" are universal, thus no third-level indices are offered thereunder.

\subsubsection{Social adaptability}


The "Social adaptability" means that EPs should be in favor of shaping proper self-awareness and social consciousness of children and help them build favorable social relationships and actively adapt to the social environment.

At least one of the following functions should be provided:

1. to help children develop self-awareness, such as better understanding themselves, building up confidence and strengthening self-control;

2. to form children's social consciousness, such as stimulating their interests of social integration, assisting them in understanding and learning social conventions;

3. to help children to build up favorable social relationships, such as learning sharing, cooperation and how to handle conflicts with other people.

Examples of EPs that don't meet the requirements for the index:

1. products that children could not control actively but only participate passively;

2. products that induce children to indulge in the virtual world and alienate themselves from the real life.

\subsubsection{Cultural Adaptability}

The "Cultural adaptability" means that EPs should root in national and local cultures, help children not only understand and adapt to local cultures but also stimulate their interests by taking advantage of local cultural resources to satisfy children's cultural background.

At least one of the following functions should be provided:

1. to allow children to get familiar with and love the local cultures;

2. to reflect national or local cultural resources;

3. to be right for children's existing cultural background;

Examples of EPs that don't meet the requirement for the index:

1. products with content of defacing or defaming national and local cultures.

\subsubsection{Aesthetic}

The "Aesthetic" means that EPs should follow aesthetic principles in design, to inspire aesthetic experiences of children that come from various fields of the products such as content, shape, color, pattern, sound, and packing

All of the following functions should be provided:

1. decent content without vulgar elements;

2. clear and artistic characters, colors and patterns;

2. proper size and shape;

\subsection{Gameplay}

The "Gameplay" means that EPs should possess game qualities and properties. Games are, in essence, activities that have operation materials, involve the active participation of children and provide them delightful emotional experiences. Different from ordinary educational and school supplies, EPs should become materials for games, stimulate children experience in game contexts, inspire their interests, and finally fulfill educational objectives through fun experience.

In view of the quintessential properties of the index "Gameplay", three categories are included, that is, "Interesting”, "Pleasurable" and "Operable". Since these three indices are universally applicable and demand no specific annotations for different stages or aspects, no third-level indices are offered thereunder.

\subsubsection{Interesting}

The "Interesting" means that EPs should be attractive in content so as to stimulate children's interests and arouse their inherent motivation. Children play with or operate EPs of their own free will, without external forces to push.

\subsubsection{Pleasurable}

The "Pleasurable" means that EPs should give children delightful experiences with happiness and satisfaction.

\subsubsection{Operable.}

The "Operable" means that EPs should provide children DIY materials and build up a DIY environment to inspire children's initiative awareness of active participation.

All of the following functions should be provided:

1. explicit operation goal;

2. appropriate operation degree of difficulty according to player's age (Please refer to Section 3.1.1 for the requirements of different stages of cognitive development);

3. clear and distinct operation rules and methods;

4. either one player or multiple player allowed;

5. repetitive operations allowed.

\section{Acknowledgement}

The research is funded by Science and Technology Department of Guangdong Province(grant number 2011B040200005).

\section{References}

[1] Kuang Wei, “Design and Study of Children's Educational Products", Thesis for Master Degree, Hubei University of Technology, 2012.

[2] Lei Li, "Developmental Psychology”, China Renmin University Press, Beijing, 2012.

[3] National Technical Committee of Standardization for Toys, "Interpretation and Implementation of National Technical Specification for Safety of Toys”, Standards Press of China, 2004.

[4] National Technical Committee of Standardization for Toys, "Interpretation and Implementation of Instructions for Consumer Goods, Part V: Toys”, Standards Press of China, 2008.

[5] Zhao Jimei, "Design and Study of Educational Products for Infants, Thesis for Master Degree, Qiqihar University, 2011.

[6] J.H.Flavell et al., translated by Deng Ciping and Liu Ming, “Cognitive Development”, East China Normal University Press, 2002.

[7] Liang Ai’ying et al., "Preliminary Study on Index System for Educational Products”, Guangdong Technology Press, [J] 2013(24).

[8] Liang Ai'ying \& Xiao Wenbin, “Survey and Thoughts on Educational Products at Domestic Science Popularization Venues”, Guangdong Technology Press, [J] 2013(22). 